\title{
Overall survival of metastatic melanoma patients treated with HD IL-2 followed by immune checkpoint blockade of the CTLA-4 or the PD-1 pathways: analysis of data on the current use of HD IL-2
}

\author{
Michael KK Wong ${ }^{1}$, Michael A Morse ${ }^{2}$, David F McDermott ${ }^{3}$, Joseph I Clark ${ }^{4}$, Howard L Kaufman ${ }^{5}$, \\ Gregory A Daniels ${ }^{6}$, Jessica C Perritt ${ }^{7}$, Hong Hua ${ }^{7}$, Sandra Aung ${ }^{7 *}$
}

From 30th Annual Meeting and Associated Programs of the Society for Immunotherapy of Cancer (SITC 2015) National Harbor, MD, USA. 4-8 November 2015

\section{Background}

HD IL-2 was FDA approved for advanced melanoma, but the data supporting its use dates to the 1990's. The PROCLAIM ${ }^{\mathrm{SM}}$ registry (http://www.proclaimregistry. com) is the largest collection of IL-2 treated patients in the US and provides real-time insights into patient survival and outcomes. Previously, we reported a median overall survival (mOS) of 20 months with a median follow-up of 37 months in metastatic melanoma $(\mathrm{mM})$ patients treated with high dose IL-2 (HD IL-2) between 2007 and 2012 from a retrospective cohort. These findings led to the hypothesis that improved mOS may have been a result of subsequent salvage therapies, including checkpoint inhibitors.

\section{Methods}

Patients must have received at least one dose of HD IL2 for this analysis. Those that received checkpoint therapy prior to HD IL-2 were excluded. Statistics and survival analysis on prospectively entered patients $(\mathrm{N}=236)$ were performed on datasets as of March 16 ${ }^{\text {th }}, 2015$.

\section{Results}

The median overall survival (mOS) for the 236 patients was 18.4 months with a median follow-up of 21.7 months. Patients were stratified into three groups; HD IL- 2 only ( $n=123)$, HD IL-2 followed by ipilimumab
(IL-2 $\rightarrow$ ipi, $\mathrm{n}=78$ ), and HD IL-2 followed by PD-1 inhibitors (IL-2 $\rightarrow$ aPD-1, $\mathrm{n}=35$ ). The majority of patients $(22$ of 35$)$ in the IL-2 $\rightarrow$ aPD-1 group had progressive disease before receiving subsequent treatment with anti-PD-1/ PD-L1-containing regimens. Patients in the HD IL-2 only, IL- $\rightarrow$ ipi, and IL- $\rightarrow$ aPD- 1 groups achieved a mOS of 14, 15.8, and 28.7 months, respectively. The estimated 12 -month survival rates were $57 \%, 64 \%$, and $97 \%$, respectively. There were $10 / 78$ (13\%) and $2 / 35$ (5.7\%) post therapy treatment-related incidences of autoimmune events in the IL- $\rightarrow$ ipi and IL- $\rightarrow$ aPD- 1 groups, respectively. No treatment related deaths were reported.

\section{Conclusions}

This is the first report of clinical data relating to HD IL2 use followed by checkpoint blockade of the PD-1 pathway. Treatment with anti-PD-1 after initial therapy with HD IL-2 had significantly prolonged survival compared to patients treated with ipilimumab. Moreover, improved survival was not observed in patients treated with follow-on ipilimumab compared to patients treated only with HD IL-2. Anti-PD-1 therapy after HD IL-2, appears to be safe and therapeutically active. These data support the concept of investigating IL-2 therapy in combination or sequence with newly developed immune checkpoint inhibitors.

${ }^{7}$ Prometheus Laboratories Inc., San Diego, CA, USA

Full list of author information is available at the end of the article 


\section{Authors' details}

${ }^{1}$ University of Southern California, Los Angeles, Los Angeles, CA, USA. ${ }^{2}$ Duke University Medical Center, Durham, NC, USA. ${ }^{3}$ The Cytokine Working Group; Division of Hematology/Oncology, Beth Israel Deaconess Medical Center, Boston, MA, USA. " Loyola University Medical Center, Division of Hematology Oncology, Maywood, IL, USA. ${ }^{5}$ Rutgers Cancer Center Institute of New Jersey, New Brunswick, NJ, USA. ${ }^{6}$ Moores Cancer Center, University of California San Diego, La Jolla, CA, USA. ${ }^{7}$ Prometheus Laboratories Inc., San Diego, CA, USA.

Published: 4 November 2015

doi:10.1186/2051-1426-3-S2-P359

Cite this article as: Wong et al: Overall survival of metastatic melanoma patients treated with HD IL-2 followed by immune checkpoint blockade of the CTLA-4 or the PD-1 pathways: analysis of data on the current use of HD IL-2. Journal for ImmunoTherapy of Cancer 2015 3(Suppl 2):P359.

\section{Submit your next manuscript to BioMed Central} and take full advantage of:

- Convenient online submission

- Thorough peer review

- No space constraints or color figure charges

- Immediate publication on acceptance

- Inclusion in PubMed, CAS, Scopus and Google Scholar

- Research which is freely available for redistribution

Submit your manuscript at www.biomedcentral.com/submit 